\title{
Linx
}

Revue des linguistes de l'université Paris X Nanterre

39 | 1998

Modèles linguistiques : convergences, divergences

\section{Centre d'Etudes et de Recherches sur la Planification Linguistique, Alternance des langues et apprentissage en contextes plurilingues}

Annie Montaut

\section{(2) OpenEdition}

Journals

Édition électronique

URL : http://journals.openedition.org/linx/930

DOI : $10.4000 / \operatorname{linx} .930$

ISSN : 2118-9692

Éditeur

Presses universitaires de Paris Nanterre

Édition imprimée

Date de publication : 15 décembre 1998

Pagination : 255-260

ISSN : 0246-8743

Référence électronique

Annie Montaut, «Centre d'Etudes et de Recherches sur la Planification Linguistique, Alternance des langues et apprentissage en contextes plurilingues », Linx [En ligne], 39 | 1998, mis en ligne le 02 juillet 2012, consulté le 22 septembre 2020. URL : http://journals.openedition.org/linx/930 ; DOI : https:// doi.org/10.4000/linx.930

Ce document a été généré automatiquement le 22 septembre 2020.

Département de Sciences du langage, Université Paris Ouest 


\title{
Centre d'Etudes et de Recherches sur la Planification Linguistique, Alternance des langues et apprentissage en contextes plurilingues
}

\author{
Annie Montaut
}

\section{RÉFÉRENCE}

Plurilinguisme nº 14, décembre 1997, Centre d'Etudes et de Recherches sur la

Planification Linguistique, Alternance des langues et apprentissage en contextes plurilingues

L'ouvrage est très inégal, incluant aussi bien des articles sans problématique ni données («alternances codiques et pratiques de classes en milieu bilingue Malgache/Français» d'Elisa Ratifoson, dont on se demande quel est l'intérêt pour le linguiste) que des articles d'une grande portée théorique et descriptive (Myers-Scotton \& Jake). Entre ces deux cas extrêmes, les contributions, en français et en anglais, qui touchent à divers aspects de l'alternance plus ou moins fondamentaux, ont presque toutes le mérite de mettre à la portée du lecteur d'excellentes données de première main, la seconde partie de l'ouvrage abordant des problèmes plus féconds pour l'étude de l'alternance comme pour la linguistique générale, peut-être parce que les terrains abordés ont déjà fait l'objet de nombreux travaux. La revue regroupe ici une partie (touchant l'immigration et les anciennes colonies) des communications présentées lors d'une rencontre à l'initiative du CREDIF sur le thème de l'alternance et de l'apprentissage à l'ENS de Fontenay-Saint Cloud (6-8 février 1997), les autres communications étant publiées ou à paraître dans les revues suivantes, dont le sommaire est donné dans l'annexe p. VII : Cahiers du Français contemporain, Etudes de Linguistique Appliquée, LIDIL (Presses Universitaires de Grenoble). 
2 Le concept d'alternance de langues, traduisant la notion de "code-switching ", est défini dans la présentation de l'ouvrage par Christine Deprez, comme «entendue au sens large, désign[ant] toute situation où un même acteur se trouve opérer à l'intérieur d'un même événement ou d'un même contexte de communication. D'un tel point de vue, le changement de codes à l'intérieur d'un même énoncé et le recours à plusieurs systèmes linguistiques, la mobilisation des références culturelles plurielles dans un domaine social donné constituent des formes d'usage entrant sous la définition» (p. 2).

3 Jean-Michel Karbarian («Emprunt, alternance balisée et modalités d'apprentissage du Français par des élèves arménophones de Marseille») précise toutefois la définition en distinguant, à la suite de Poplack, l'alternance ordinaire ou fluide et l'alternance «balisée» ou «saillante» et constate dans son étude sur l'école Hamaskaïne que le recours au simple emprunt de mots signale une moindre familiarité que l'alternance et surtout l'alternance balisée (dominante à partir de sept ans de scolarisation en France), mais est néanmoins plus fréquent en situation informelle que formelle, quel que soit le degré de sécurité en français chez les arménophones.

4 L'article de Didier de Robillard sur le français régional de l'Ile Maurice à travers la correction de copies met en doute la pertinence du caractère xénique comme facteur de rejet pour les témoins correcteurs (les élèves), lesquels assimilent seulement un tiers des régionalismes à des fautes, indice d'une certaine sécurité linguistique de la communauté dans ses usages propres. On peut s'étonner de ne pas voir figurer dans la bibliographie d'un article qui accorde toute une section à la «situation sociolinguistique de l'Ile Maurice» l'ouvrage pourtant en français de Peter Stein (Connaissance et emploi des langues à l'Ille Maurice, Helmut Busk Verlag, Hamburg, 1982).

5 Leo Van Lier («Critical awareness and language use in multilingual classrooms : a social interactionist perspective») propose une ouverture sociologique à la politique linguistique fort sensée --qui a déjà été proposée dans les années quatre-vingt et même soixante-dix en contexte indien (D.P. Pattanayak, Language Curriculum, 1972, et Language, Education and Culture, 1991, CIIL, Mysore, Inde). A partir d'une expérience californienne visant à faire reconnaître le statut de l'«ebonics» (ebony + phonetics : une variété bien particulière d'anglais parlé par les noirs, ses spécificités étant liées au Niger-Congo), il plaide généreusement en faveur d'une conception de l'école comme communauté discursive et lieu d'interaction, capable de voir le multilinguisme et le multidialectalisme comme une ressource à exploiter plus que comme un problème à surmonter.

6 Suivent trois études sur les phénomènes d'alternance en milieu algérien, qui ouvrent, avec de riches données de première main, sur de véritables problèmes intéressant le linguiste général aussi bien que le spécialiste du «code-switching». Cherifa Ghettas ("Quelques éléments de réponse sur le passage de l'arabe dialectal à l'arabe standard en classe de langue chez l'enfant algérien d 5 à 7 ans») reprend l'idée de Taleb-Ibrahimi d'un continuum linguistique plutôt que d'une véritable diglossie telle que la décrivent Marçais et Ferguson, et montre sobrement que des trois stratégies observables sur son corpus (continuité et non rupture entre vernaculaire et langue de l'école, susbstitution, enfermement dans la reproduction des schémas stéréotypés conformes à la norme), la seconde, observée chez les 7 ans, est un effet pervers de la représentation diglossique que véhicule la scolarisation, et que la première (plus fréquente chez les plus jeunes) devrait être encouragée par une prise de conscience réelle des acquis antérieurs de l'enfant et de leur pertinence dans le cadre de l'apprentissage de la norme littérale, 
ainsi que par la prise de conscience chez l'enfant de ce continuum. Notons que Bronckart 1985 cité dans le texte ne figure pas en bibliographie et que le texte est déparé par les erreurs typographiques, orthographiques et stylistiques.

7 La présentation de Dominique Caubet («Alternance des codes au Maghreb : pourquoi le français est-il arabisé ?») a entre autres le mérite de porter sur les aspects phonétiques et intonatifs, souvent laissés pour compte dans le domaine de l'alternance en milieu maghrébin, trait qu'elle inclut dans dans le gouvernement de la langue enchâssée par la langue matrice, au moins à titre de règle optionnelle. Après une mise en place claire et bien documentée du code-switching en Algérie et des français d'Algérie, elle analyse à travers la prestation d'un comédien populaire Fellag la classe des locuteurs "caméléons» (capables d'adopter selon les contextes la variété standard élevée, standard ordinaire, ou algérianisée du français), et joint un extrait du spectacle Djurdjurassic Park intéressant à de nombreux titres. Elle conclut que la prononciation maghrébinisée du français est un des constituants fondamentaux de la communauté linguistique réelle (en tant que distincte de la communauté imaginaire), et fait intrinsèquement partie $\mathrm{du}$ processus de code-switching (largement inconscient), le «code-switcher» ayant besoin de se sentir en sécurité pour ne pas être inhibé par la stigmatisation (y compris l'auto-sigmatisation) sanctionnant ce mode de communication.

8 Amina Bensalah («L'effet loupe des formes mêlées et discours rapportés») précise dès son titre, explicité dans les premières sections (où ni Gadet ni Labov, cités dans le texte, n'apparaissent en bibliographie) que l'alternance dans les discours rapportés est un poste d'observation privilégié pour des phénomènes plus généraux observables dans les interlocutions de «pseudo-monolinguisme». Cette idée me semble extrêmement féconde et encore largement à explorer, rendant compte d'écritures et de pratiques typiquement hybrides, en prise sur un monde de plus en plus «multiculturel», qu'il s'agisse de ce que Rushdie appelle la «mongrelization» ou Glissant la créolisation. Dans le cas particulier de l'arabe en alternance avec le français, les conclusions révèlent qu'à l'oral le discours direct est très rarement la reprise des paroles authentiques d'autrui, la langue de leur re-formulation ne correspondant pas forcément à la langue du locuteur cité, mais à une rhétorique conversationnelle où le "pôle de la réception et le dessein dicursif l'emportent sur l'authenticité des formes discursives rapportées», facteur qui distingue crucialement le discours rapporté oral de son correspondant écrit, qu'il y ait ou non alternance de langue. En outre, le jeu de l'alternance y apparaît comme «la trace d'une attitude méta-évaluative» (les commentaires personnels et les faits étant souvent rapportés dans des langues différentes, dans une sorte de dénivellement énonciatif, comme du reste dans les segments non rapportés), sans discours métalinguistique, jouant en cela un rôle comparable à la dimension suprasegmentale de tout discours oral.

9 Le texte d'Elena Silvestri (" '...This is regular conversation» : autocommentaires dans une conversation familiale bilingue», dans le contexte de l'immigration italophone au Canada anglophone) a des ambitions plus modestes, mais montre aussi que l'alternance sert, entre autres, à évaluer ses propres productions comme celles d'autrui, et ce, dans un milieu où il n'y a pas de langue dominante, l'anglais étant celle des enfants mais l'italien celle des parents. Non seulement le corpus est amusant, mais l'auteur montre bien que l'alternance, dans ses utilisations ludiques, est créative et dynamisante. 

de cette sympathique famille, si l'article suivant, de Sylvia Milian Hita («Code Switching and Face Threatening Acts: multifunctionality and ambiguity of code switching in family and peer interactions») ne présentait une famille portoricaine bilingue (espagnol-anglais) qui utilise l'alternance d'une façon beaucoup moins conviviale. L'auteur utilise des concepts intéressants, empruntés à Myers-Scotton et Brown \& Levinson, comme le Markedness Model, et le Politeness Model, fondé sur le rôle et l'ensemble des obligations liées à la position dans le groupe social, et prend appui sur la logique conversationnelle de Grice, montrant au passage que les modèles ici pertinents traitent les implicatures et les inférences comme relevant de la manière dont le code et la structure du message instancient les relations sociales, et non de la seule logique de ces relations. Analysant les relations entre alternance et actes menaçant l'image publique que veut avoir le locuteur ou l'interlocuteur (stratégies visant à sauver la face ou à faire perdre face), l'auteur démontre, contre Brown \& Levinson, que le code switching n'est pas seulement une stratégie de politesse servant à euphémiser une menace potentielle, mais est utilisé dans la manipulation des stratégies de menace comme dispositif d'aggravation, et qu'en lui-même le code switching constitue une menace à l'image d'autrui s'il est employé pour encoder une telle menace (usage de l'anglais dans le couple hispanophone quand la situation s'envenime). Même dans une situation linguistique où l'aternance est en soi non marquée, contrairement à ce qu'avance Myers-Scotton, chaque occurrence d'alternance peut être socialement signifiante à un moment donné, et notamment être indice d'une intention de négociation, ou d'un changement de l'ensemble des devoirs et obligations.

11 Le long article enfin de Carol Myers-Scotton \& Janice L. Jake qui clôt l'ouvrage («Codeswitching and the nature of lexical entries», pp. 219-46) est une véritable contribution à la linguistique théorique, qui intéressera le typologue et le comparatiste aussi bien que le générativiste formaliste. L'auteur utilise l'occurrence ou la non occurrence d'îlots en langue enchâssée («embedded language islands») dans les verbes des complétives et les groupes nominaux pour montrer que les entrées lexicales ne peuvent pas être définies sur la seule base de leur réalisation de surface, mais qu'il faut faire intervenir la notion de lemme («lemma», entité abstraite dans le lexique mental) contenant toute l' information nécessaire pour compléter le constituant où figure le morphème, et qui peut être assigné structurellement ou bien à un niveau plus abstrait («indirectly elected»). Si une entrée lemmatique en langue enchâssée (EL) est congruente avec son correspondant dans la langue matrice (ML), le constituant résultant est bien formé, comportant un lexème EL et des morphèmes systémiques (grammaticaux) ML. L'occurrence d'îlots atypiques EL, entièrement consituées de morphèmes EL, lexicaux et grammaticaux, suggère la non congruence des deux systèmes lemmatiques impliqués dans l'alternance. Ainsi, les îlots atypiques en anglais dans une phrase arabe quand il s'agit du verbe dans la projection du complémenteur (espérer que $V$, essayer de $V$ ) présentent un verbe non fléchi, alors que le verbe devrait porter des marques aspectuelles et temporelles arabes comme c'est le cas dans d'autres emprunts de verbe anglais en phrase simple. Cette dissymétrie dans le comportement $\mathrm{du}$ verbe dans CP et ailleurs vient de ce que dans la projection du complémenteur, le schéma d'analyse est déterminé par l'arabe, et qu'en arabe temps et aspect sont des morphèmes activés en même temps que les autres traits conceptuels du verbe, alors qu'en anglais ils sont activés plus tard, au niveau structurel (positionnel) et non mental, la sélection du verbe impliquant en arabe IP dans son ensemble et pas seulement VP. 
D'où l'occurrence de l'îlot anglais, incluant les morphèmes grammaticaux sous INFL, comme correspondant au verbe arabe, dans la projection du complémenteur. Le comportement des noms espagnols dans une phrase anglaise produit des asymétries semblables. Alors qu'on s'attendrait à des constituants mixtes ou à l'occurrence d'îlots à égalité, il y a très peu d'îlots NP quand la langue matrice est romane (la potato, el half chicken). Les auteurs expliquent cette asymétrie par la différence de fonctionnement des traits phi, activés plus tôt, à un plus haut niveau d'abstraction en espagnol («indirectly elected»: en dépendent l'assignement du cas et la cliticisation) qu'en anglais («structurally-assigned»): c'est pourquoi les déterminants viennent de l'espagnol quand l'espagnol est la langue matrice qui détermine le schéma d'analyse du groupe nominal. L'asymétrie italien/allemand (liée à la différence entre langue 'prodrop' ou à sujet nul et langue 'non pro-drop') fait l'objet d'une dernière analyse sur ces mêmes principes, et l'auteur conclut sur la nature de la structure linguistique, qui implique des degrés d'abstraction différents dans la structure lexicale: ce qui est saillant à tel niveau dans une langue donnée ne l'est pas forcément au même niveau dans une autre langue, ce que vise à prendre en compte la notion de lemme. 